\title{
Ginsenoside Rg1 Attenuates Neuroinflammation Following Systemic Lipopolysaccharide Treatment in Mice
}

\author{
Jung-Won Shin", Sun-Ho Ma\#, Ju-Won Lee, Dong-Kyu Kim, Kyuho Do, Nak-Won Sohn* \\ Department of Oriental Medical Science, Graduate School of East-West Medical Science, Kyung Hee University
}

\begin{abstract}
Objectives : Neuroinflammation is characterized by microglial activation and the expression of major inflammatory mediators. The present study investigated the inhibitory effect of ginsenoside $\operatorname{Rg} 1$ (GRg $)$, a principle active ingredient in Panax ginseng, on pro-inflammatory cytokines and microglial activation induced by systemic lipopolysaccharide (LPS) treatment in the mouse brain tissue.

Methods : Varying doses of $\mathrm{GRg}_{1}$ was orally administered (10, 20, and $30 \mathrm{mg} / \mathrm{kg}$ ) $1 \mathrm{~h}$ before the LPS injection (3 $\mathrm{mg} / \mathrm{kg}$, intraperitoneally). The mRNA expression of pro-inflammatory cytokines in the brain tissue was measured using the quantitative real-time PCR method at $4 \mathrm{~h}$ after the LPS injection, Microglial activation was evaluated using western blotting and immunohistochemistry against ionized calcium binding adaptor molecule 1 (Iba1) in the brain tissue. Cyclooxigenase-2 (COX-2) expressions also observed using western blotting and immunohistochemistry at $4 \mathrm{~h}$ after the LPS injection, In addition, double-immunofluorescent labeling of tumor necrosis factor $-\alpha(\mathrm{TNF}-\alpha)$ and $\mathrm{COX}-2$ with microglia and neurons was processed in the brain tissue.

Results : $\operatorname{GRg}_{1}(30 \mathrm{mg} / \mathrm{kg})$ significantly attenuated the upregulation of TNF- $\alpha$, interleukin (IL) $-1 \beta$ and IL-6 mRNA in the brain tissue at $4 \mathrm{~h}$ after LPS injection. Morphological activation and Iba1 protein expression of microglia induced by systemic LPS injection were reduced by the $\mathrm{GRg}_{1}(30 \mathrm{mg} / \mathrm{kg})$ treatment. Upregulation of COX-2 protein expression in the brain tissue was also attenuated by the $\mathrm{GRg}_{1}(30 \mathrm{mg} / \mathrm{kg})$ treatment.

Conclusion : The results suggest that $\mathrm{GRg}_{1}$ is effective in the early stage of neuroinflammation which causes neurodegenerative diseases.
\end{abstract}

Key words : Ginsenoside $\mathrm{Rg}_{1}$, Neuroinflammation, Pro-inflammatory Cytokines, Microglia activation, Cyclooxygense-2

\section{Introduction}

Neuroinflammation is the inflammation of the central nervous system (CNS), and it is considered a mediator of secondary damage. It is characterized by microglia activation and expression of major inflammatory mediators, including pro-inflammatory cytokines and cyclooxygenase $(\mathrm{COX})^{1,2)}$. Neurodegenerative diseases, including Alzheimer' $\mathrm{s}$ disease (AD) and Parkinson' $\mathrm{s}$ disease (PD), are characterized by chronic inflammatory responses in the $\mathrm{CNS}^{3)}$. Microglia, the resident immune cells in the CNS, are key cellular elements of the acute neuroinflammatory response and are the primary sources of pro-inflammatory cytokines detected in the brain $^{3)}$. COX-2 is responsible for propagating the inflammatory response and is thus considered the best target for anti-inflammatory drugs ${ }^{2}$. Therefore, determining the modulatory effects of various drugs

\footnotetext{
*Corresponding Author : Nak-Won Sohn. Department of Oriental Medical Science, Graduate School of East-West Medical Science, Kyung Hee University 1732 Deogyeong-daero, Giheung-gu, Yongin 446-701, Republic of Korea

Tel : 031-201-2747 · Fax: 031-204-6832 ·E-mail : sohnnw@khu.ac.kr

\#First author : Jung-Won Shin. Department of Oriental Medical Science, Graduate School of East-West Medical Science, Kyung Hee University 1732 Deogyeong-daero, Giheung-gu, Yongin 446-701, Republic of Korea

· Tel : 031-201-2747 ·Fax: 031-204-6832 ·E-mail : shinarago@khu.ac.kr

Sun-Ho Ma. Department of Oriental Medical Science, Graduate School of East-West Medical Science, Kyung Hee University 1732 Deogyeong-daero, Giheung-gu, Yongin 446-701, Republic of Korea

· Tel : 031-201-2747 · Fax: 031-204-6832 ·E-mail : doinsarang@hanmail.net

· 접수 : 2013년 10월 23일 ·수정 : 2013년 11월 09일 · 채택 : 2013년 11월 12일
} 
on neuroinflammation is critical to developing therapeutic approaches for acute and chronic CNS disorders.

Ginsenoside $\mathrm{Rg}_{1}\left(\mathrm{GRg}_{1}\right)$ is an active ingredient in Ginseng, the root of Panax ginseng C.A. Meyer, Araliaceae $^{4}$. Ginseng is one of the most widely investigated medicinal herb and exerts ameliorating effects on the central nervous system disorders such as stroke, $\mathrm{AD}, \mathrm{PD}$, and amyotrophic lateral sclerosis ${ }^{5)}$. Ginseng also alleviates neuroinflammatory processes through regulation of neurotransmitter release and pro-inflammatory mediators ${ }^{5)}$. Ginsenosides can be classified into 3 categories: the panaxadiol group (e.g., $\mathrm{Rb}_{1}, \mathrm{Rb}_{2}$, and $\mathrm{Rg}_{3}$ ), the panaxatriol group (e.g., $\mathrm{Rg}_{1}, \mathrm{Rg}_{2}$, and $\mathrm{Rh}_{1}$ ), and the oleanolic acid group (e.g., Ro $)^{4)}$. The characteristic actions of each group are different from other groups ${ }^{4)}$. In particular, the protective effects of panaxatriol $\mathrm{GRg}_{1}$ against neurodegeneration are well studied ${ }^{6-13)}$. With regard to neuroinflammation, panaxadiol ginsenoside $\mathrm{Rg}_{3}$ demonstrated anti-neuroinflammatory effects ${ }^{14)}$. Studies of $\mathrm{GRg}_{1}$ have demonstrated anti-diabetic activity ${ }^{15)}$, hematopoietic function ${ }^{16)}$, estrogenic effects $^{17)}$, and anti-inflammatory effects ${ }^{18)}$. Recently, in vitro evidence has emerged that $\mathrm{GRg}_{1}$ may play a role in protection against neurodegenerative conditions ${ }^{6-9)}$. In these studies, GRg $_{1}$ has been shown to attenuate beta-amyloid $(\mathrm{A} \beta)$ generation as a peroxisome proliferator-activated receptor-gamma agonist ${ }^{6)}$, cause tau phosphorylation in $\mathrm{A} \beta$-stimulated THP-1 cells (human acute monocytic leukemia cell line $)^{7)}$, induce neuronal damage in hypoxic ischemic injury ${ }^{8)}$, and protect the dopaminergic cell line against 6-hydroxydopamine-induced toxicity ${ }^{9}$. Moreover, in animal studies, $\mathrm{GRg}_{1}$ reduced $\mathrm{A} \beta$ accumulation and improved cognitive impairments in an ovariectomized rat model of $\mathrm{AD}^{10)}$, in transgenic $\mathrm{AD}$ mice ${ }^{11)}$, and in senescence-accelerated mouse prone-/8 mice ${ }^{12)}$. Furthermore, GRg ${ }_{1}$ protected dopaminergic neurons against injury to the nigrostriatal dopaminergic pathway and ameliorated dysfunctional behaviors ${ }^{13)}$. Overall, these reports indicate that $\mathrm{GRg}_{1}$ can play a modulatory role in neuroinflammation that causes neurodegenerative diseases.

Microglial activation and pro-inflammatory mediators play a major role in the neuroinflammatory mechanisms of the $\mathrm{CNS}^{19,20)}$. There are few in vivo studies investigating the effect of $\mathrm{GRg}_{1}$ on microglial activation in the brain tissue except a report from $\mathrm{Hu}$ et $\mathrm{al}^{21)}$. Therefore, to better understand the anti-neuroinflammatory effects of $\mathrm{GRg}_{1}$, the present study investigated the effect of $\mathrm{GRg}_{1}$ on microglial activation, which a major character in neuroinflammation with pro-inflammatory cytokine and COX-2 expression, in the brain tissue of LPS-treated mice.

\section{Materials and Methods}

\section{Materials}

\section{1) Animals}

Male C57BL/6 mice (25-28 g; Nara Biotechnology, Korea) were used for this study. All animal protocols were approved by the Ethics Committee for the Care and Use of Laboratory Animals at Kyung Hee University. The animals were housed in plastic cages at a constant temperature $\left(22 \pm 2{ }^{\circ} \mathrm{C}\right)$ and humidity $(55 \pm 10 \%)$ under $12-\mathrm{h}$ light-dark conditions. The animals were allowed free access to food and water before the experiment.

\section{2) Reagents}

$\mathrm{GRg}_{1}\left(\mathrm{C}_{42} \mathrm{H}_{72} \mathrm{O}_{14}\right.$; molar weight, 801.01; Fig. 1) was purchased from LKT Laboratories (Saint Paul, MN, USA). LPS (from Escherichia coli 055:B5) was purchased from Sigma-Aldrich (St. Louis, MO, USA). Rabbit anti-ionized calcium binding adaptor molecule 1 (Iba1) antibodies (\#16-20001, \#019-19741) were purchased from Wako Pure Chemical Industries (Osaka, Japan). Goat anti-tumor necrosis factor- $\alpha$ $(\mathrm{TNF}-\alpha)$ antibody was purchased from Santa Cruz Biotechnology (Santa Cruz, CA, USA). Rabbit anti-COX-2 antibody for immunohistochemistry was purchased from Cayman Chemical (Ann Arbor, MI, USA). Goat anti-COX-2 antibody for western blotting was purchased from Abcam (Cambridge, UK). Mouse anti-actin antibody was purchased from Chemicon International (Temecula, CA, USA). Mouse anti-neuronal nuclei (NeuN) antibody and secondary antibodies were purchased from Millipore (Temecula, CA, USA). Cy2-conjugated donkey anti-mouse or donkey anti-rabbit immunoglobulin G (IgG), and Cy3-conjugated donkey anti-mouse IgG were purchased from Jackson ImmunoReseach Laboratories (West Grove, PA, USA). The other chemicals and reagents used were of high quality and obtained from various commercial sources.

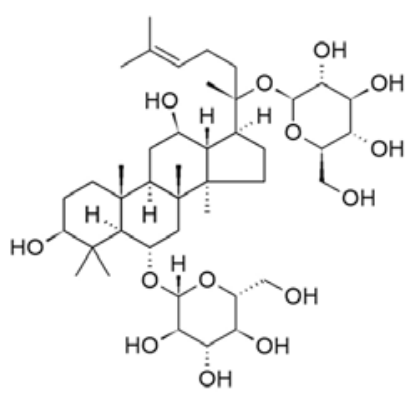

Fig. 1. Chemical structure of ginsenoside Rg1. 


\section{Methods}

\section{1) Experimental groups}

Mice were randomly divided into 5 groups. The normal group (Normal) was allowed free access to food and water without any treatment. The control group (LPS) was intraperitoneally (i.p.) injected with a single dose of LPS $(3 \mathrm{mg} / \mathrm{kg})$ and received the vehicle (normal saline) orally $1 \mathrm{~h}$ before the LPS injection.

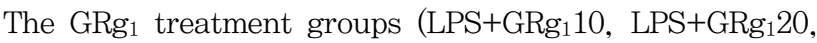
and $\left.\mathrm{LPS}+\mathrm{GRg}_{1} 30\right)$ were administered an oral dose of $\mathrm{GRg}_{1}(10,20$, or $30 \mathrm{mg} / \mathrm{kg}$, respectively, dissolved in normal saline) $1 \mathrm{~h}$ prior to LPS injection. Immunohistochemistry studies were processed separately from the polymerase chain reaction (PCR) and western blotting studies in different animals. A total of 48 mice (6 mice per group) were used in this study.

\section{2) Real-time PCR measurement}

The mRNA expression of $\mathrm{TNF}-\alpha$, interleukin (IL) -1 $\beta$, and IL-6 in the brain tissue was measured using the quantitative real-time PCR method. At $4 \mathrm{~h}$ after the LPS injection, mice were sacrificed by decapitation and the brain tissue was rapidly dissected. Total RNA was extracted from the samples with Trizol (Qiagen, Germany) according to the manufacturer' s protocol. One microgram of total RNA was transcribed into DNA by using an iScript cDNA synthesis Kit (Bio-Rad, USA). After reverse transcription, quantitative real-time PCR was performed using preoptimized primer/probe mixture with the iQ SYBR Green Supermix kit (Bio-Rad, USA) and the CFX 96 Real-Time PCR Detection System (Bio-Rad, USA). Primer sequences for the analyzed genes are as follows: (1) $\mathrm{TNF}-\alpha$; forward, 5'-TGA GAA GTT CCC AAA TGG C-3'; reverse, 5'-GCT ACA GGC TTG TCA CTC-3'; (2) IL-1 $\beta$; forward, 5'-TGA GCA CCT TCT TTT CCT TCA-3'; reverse, 5'-TTG TCT AAT GGG AAC GTC ACA C-3' ; (3) IL-6; forward, 5' -AGA CTT CAC AGA GGA TAC CA-3'; reverse, 5’-GCA TCA TCG TTG TTC ATA CA-3'; (4) $\beta$-actin; forward, 5'-TTT CCA GCC TTC CTT GGG TAT G-3' ; reverse, 5'-CAC TGT GTT GGC ATA GAG GTC TTT AC-3'. The relative difference in expression between samples was represented using cycle time values normalized in reference to the measurement of the housekeeping gene $\beta$-actin. The sample values represent $\mathrm{x}$-fold differences from a sample from the Normal group (given as a designated value of 1) within the same experiment.

\section{3) Western blotting}

The brain tissue was homogenized and sonicated on ice in lysis buffer (50 mM Tris-HCl, pH 8.0; $150 \mathrm{mM}$
$\mathrm{NaCl} ; 1 \%$ Triton $\mathrm{X}-100 ; 0.5 \%$ sodium deoxycholate; $0.1 \%$ sodium dodecyl sulfate (SDS); $1 \mathrm{mM}$ EDTA; and $1 \%$ protease inhibitor cocktail; Sigma, USA). After centrifugation, the supernatant was collected and assayed for protein concentration by using the Bradford method. The lysates containing $50 \mathrm{\mu g}$ proteins were fractionated by SDS-10\% polyacrylamide gel electrophoresis, and then subjected to western blot analysis. The primary antibodies used in this study were rabbit anti-Iba1 antibody (\#016-20001; Wako, Japan), goat anti-COX-2 antibody (ab23672; Abcam, UK), and mouse anti- $\beta$-actin antibody (MAB1501; Chemicon, USA).

\section{4) Immunohistochemistry}

Immunohistochemistry was used to observe microglia, which were immuno-labeled with Iba1 as a microglia marker, and COX-2 in the brain tissue. At $4 \mathrm{~h}$ after the LPS injection, the mice were deeply anesthetized and perfused transcardially with $0.05 \mathrm{M}$ phosphate-buffered saline (PBS) containing 4\% paraformaldehyde. The brain was removed and postfixed in the same perfusion solution overnight at $4{ }^{\circ} \mathrm{C}$. Coronal sections of $30 \mathrm{\mu m}$ thickness were made using a freezing microtome (Leica, 2800N, Germany). The brain sections were stained by a free-floating 3,3 -diaminobenzidine (DAB) reaction. The sections were rinsed with $0.05 \mathrm{M}$ PBS and incubated for $15 \mathrm{~min}$ in $1 \%$ hydrogen peroxide PBS at room temperature. The sections were incubated overnight at $4{ }^{\circ} \mathrm{C}$ with primary antibodies against COX-2 (1:200, 160106; Cayman, USA) and Iba1 (1:500, \#019-19741; Wako, Japan). The sections were then incubated with biotinylated anti-mouse secondary antibody (1:200, Millipore, USA) for $1 \mathrm{~h}$ at room temperature, after which the avidin-biotin complex (Vector Laboratories, USA) method was carried out with peroxidase coupling in a mixture containing $0.05 \%$ DAB (Sigma-Aldrich, USA) and $0.03 \% \mathrm{H}_{2} \mathrm{O}_{2}$ for $2^{-5} \mathrm{~min}$. Primary antibodies against COX-2 (1:200, 160106; Cayman, USA), NeuN (1:200, MAB377; Millipore, USA), and Iba1 (1:500, \#019-19741; Wako, Japan) were used for double-immunofluorescent labeling of COX-2, neuron, and microglia, respectively. Moreover, a primary antibody against TNF- $\alpha$ (1:200, sc-1349; Santa Cruz, USA) was used for the double-immunofluorescent labeling of microglia. The anti-rabbit or anti-mouse Cy2 and Cy3 (Jackson ImmunoResearch, USA) was used as a secondary antibody. Images of the DAB-colorized brain sections were captured using a light microscope (BX51; Olympus, Japan) equipped with a CCD camera (DP70; Olympus, Japan) and the 
double-fluorescent-labeled images were captured using confocal laser-scanning microscopy (LSM 510 META; Carl Zeiss, Germany).

\section{5) Analysis of $\mathrm{Iba} 1$ and COX-2 immuno-positive} cells

Immunohistochemistry stained sections were used to analyze the number of immuno-positive cells. The Iba1 immuno-positive microglia and COX-2 expressed cells counts were analyzed using ImageJ software (Ver. 1.44p, NIH, USA). Four sections and 4 fields per section were chosen for the analysis of each mouse. Data were normalized to the same area $\left(10^{5}\right.$ $\mu \mathrm{m}^{2}$ ), and the mean values for the 4 sections in each mouse were used for the statistical analysis.

\section{6) Statistical analysis}

All data in this study are presented as means \pm SEM. Differences between groups were evaluated using Student' s t-test. A probability value less than 0.05 was used to indicate a significant difference.

\section{Results}

\section{Pro-inflammatory cytokine mRNA}

\section{expression in the brain tissue}

Quantitative real-time PCR measurements of brain $\mathrm{TNF}-\alpha, \mathrm{IL}-1 \beta$, and IL- 6 mRNA were conducted at 4 $\mathrm{h}$ after the LPS injection. The LPS injection increased brain levels of TNF- $\alpha$ mRNA by $\sim 80-$ fold $(80.3 \pm 6.2$ fold), while the $\mathrm{GRg}_{1}$ treatment significantly attenuated TNF- $\alpha$ mRNA levels in the brain tissue at

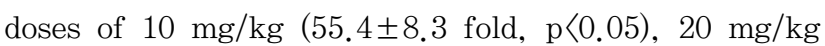
$(51.3 \pm 8.7$ fold, $\mathrm{p}<0.05)$, and $30 \mathrm{mg} / \mathrm{kg} \quad(39.0 \pm 9.1$ fold, $p<0.01)$ compared to the LPS group. The inhibitory effect of $\mathrm{GRg}_{1}$ on brain $\mathrm{TNF}-\alpha$ mRNA expression followed a dose-dependent pattern (Fig. 2-A). LPS also increased brain IL-1 $\beta$ mRNA expression by $\sim 110$-fold (109.4 \pm 14.1 fold), while $\mathrm{GRg}_{1}$ treatment significantly attenuated IL-1 $\beta$ mRNA expression in the brain tissue at a dose of $30 \mathrm{mg} / \mathrm{kg}$ (51.2 \pm 7.1 fold, $\mathrm{p}<0.01)$ compared to the LPS group. The brain levels of $\mathrm{IL}-1 \beta$ mRNA in the 10 and 20 $\mathrm{mg} / \mathrm{kg} \quad \mathrm{GRg}_{1}$ treatment groups were not significantly different from that of the LPS group (Fig. 2-B). Similarly, the brain IL-6 mRNA level was increased by $\sim 210$-fold (207.9 \pm 31.1 fold) by LPS injection, and $\mathrm{GRg}_{1}$ treatment significantly attenuated IL-6 mRNA expression in the brain tissue at a dose of $30 \mathrm{mg} / \mathrm{kg}$ (98.6 \pm 12.3 fold, $\mathrm{p}<0.01)$ compared to the LPS group
(Fig. 2-C). Overall, the increases in the levels of $\mathrm{TNF}-\alpha$, IL-1 $1 \beta$, and IL-6 mRNA in the brain tissue following systemic LPS injection were consistently reduced by the $30 \mathrm{mg} / \mathrm{kg} \mathrm{GRg}_{1}$ treatment.
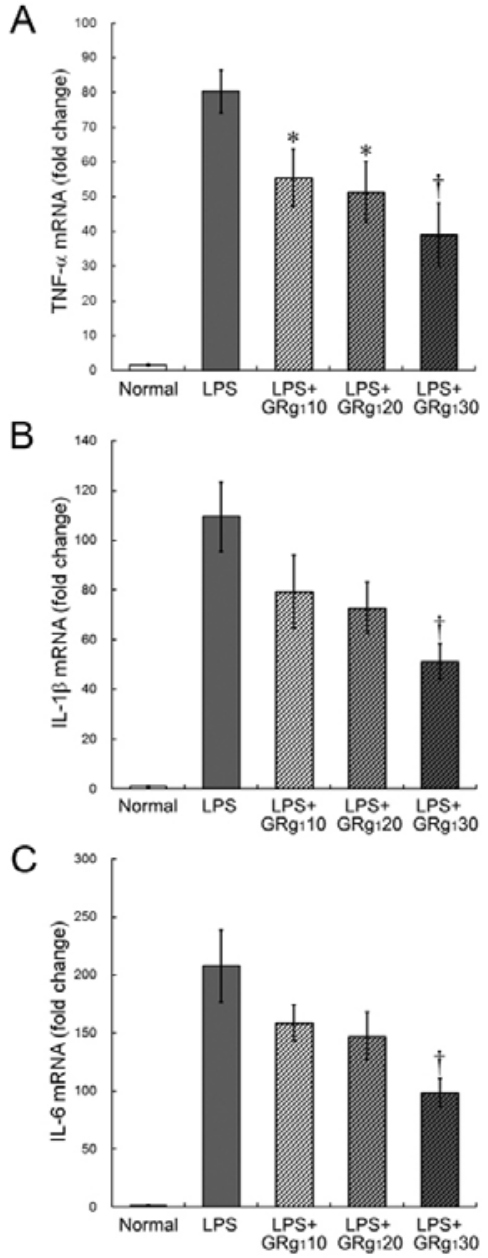

Fig. 2. The effects of $\mathrm{GRg}_{1}$ on TNF- $\alpha, \|-1 \beta$, and $\|-6$ mRNA in the brain tissue. (A) $\mathrm{GRg}_{1}$ treatment attenuates brain TNF- $\alpha$ mRNA at all doses of 10,20 , and $30 \mathrm{mg} / \mathrm{kg}$. (B) GRg ${ }_{1}$ treatment attenuates brain $\mathbb{L}-1 \beta \mathrm{mRNA}$ at a dose of $30 \mathrm{mg} / \mathrm{kg}$. (C) $\mathrm{GRg}_{1}$ treatment attenuates brain $\mathrm{LL}-6 \mathrm{mRNA}$ at a dose of $30 \mathrm{mg} / \mathrm{kg}$. Pro-inflammatory cytokine mRNA in the brain tissue was measured with quantitative real-time PCR $4 \mathrm{~h}$ after LPS injection, Data are represented by mean \pm SEM ( $n=6$ in each group). Statistical significances are based on comparison to the LPS group (*, p $<0.05 ; \dagger, p<0.01)$ using Student' $s$ t-test.

\section{Microglial activation in the brain tissue}

The effects of $\mathrm{GRg}_{1}$ on microglial activation following systemic LPS injection were tested by quantifying Iba1 protein in the brain tissue at $4 \mathrm{~h}$ after the LPS injection by using western blot analysis and immunohistochemistry with an Iba1 antibody. LPS increased the percentage of Iba1 expression in the brain tissue (153.2 $\pm 10.5 \%)$, while GRg $_{1}$ treatment significantly attenuated the percentage increase of Iba1 expression in the brain tissue at a dose of $30 \mathrm{mg} / \mathrm{kg}$ 
(112.9 $\pm 10.6 \%, p<0.05)$ compared to the LPS group. The brain levels of Iba-1 expression in the 10 and 20 $\mathrm{mg} / \mathrm{kg} \mathrm{GRg}_{1}$ treatment groups were not significantly different from that of the LPS group (Fig. 3).

Immunohistochemistry with the Iba1 antibody was performed on tissue from mice treated with a 30 $\mathrm{mg} / \mathrm{kg}$ dose of $\mathrm{GRg}_{1}$ because $30 \mathrm{mg} / \mathrm{kg}$ of $\mathrm{GRg}_{1}$ was the most effective dose for the inhibition of cytokine and Iba1 expression. The injection of LPS injection activated microglia in the cerebral cortex of the brain, as evidenced by their increased cell size, irregular shape, thickened and shortened processes, and intense Iba1 immunostaining (Fig. 4, LPS vs. Normal). However, $\mathrm{GRg}_{1}$ treatment attenuated the morphological changes associated with the activated form of microglia in all the brain regions from mice treated with LPS (Fig. 4, LPS+GRg 130 vs. LPS). To determine the effect of $\mathrm{GRg}_{1}$ on microglial activation, the number of Iba1-immunostained microglia in a standard area $\left(10^{5}\right.$ $\mu^{2}$ ) was counted. The LPS group had 60.2 \pm 3.0 cells $/ 10^{5} \mu^{2}$, whereas the LPS+GRg 130 group had $51.3 \pm 2.4$ cells $/ 10^{5} \mu \mathrm{m}^{2}(\mathrm{p}<0.05)$ (Fig. 4). These results indicate that $\mathrm{GRg}_{1}$ attenuated microglial activation in the brain induced by systemic LPS injection. In addition, double-immunofluorescent labeling of Iba1 and $\mathrm{TNF}-\alpha$ showed that $\mathrm{TNF}-\alpha$-expressing cells in the brain were co-localized mostly with the microglia (Fig. 5, Iba1+TNF- $\alpha$ ). This result indicates that TNF$\alpha$ is mainly expressed on microglia in the brain tissue following systemic LPS injection.
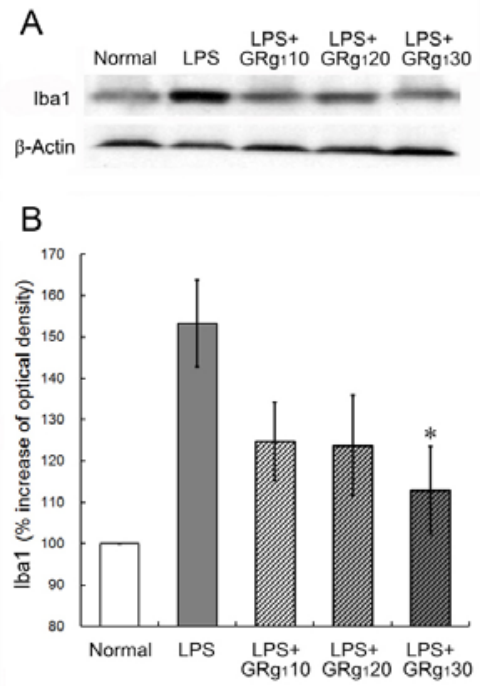

Fig. 3. The effect of $\mathrm{GRg}_{1}$ on Iba1 expression in the brain. (A) Representative western blots illustrate differences in the bands of Iba1. (B) The LPS treatment upregulated Iba1 protein, while the $\mathrm{GRg}_{1}$ treatment significantly attenuated upregulation of Iba1 protein at a dose of $30 \mathrm{mg} / \mathrm{kg}$. Data are represented by mean \pm SEM ( $n=6$ in each group). Statistical significances are based on comparison to the LPS group (*, $p<0.05$ ) using Student' $s$ t-test.
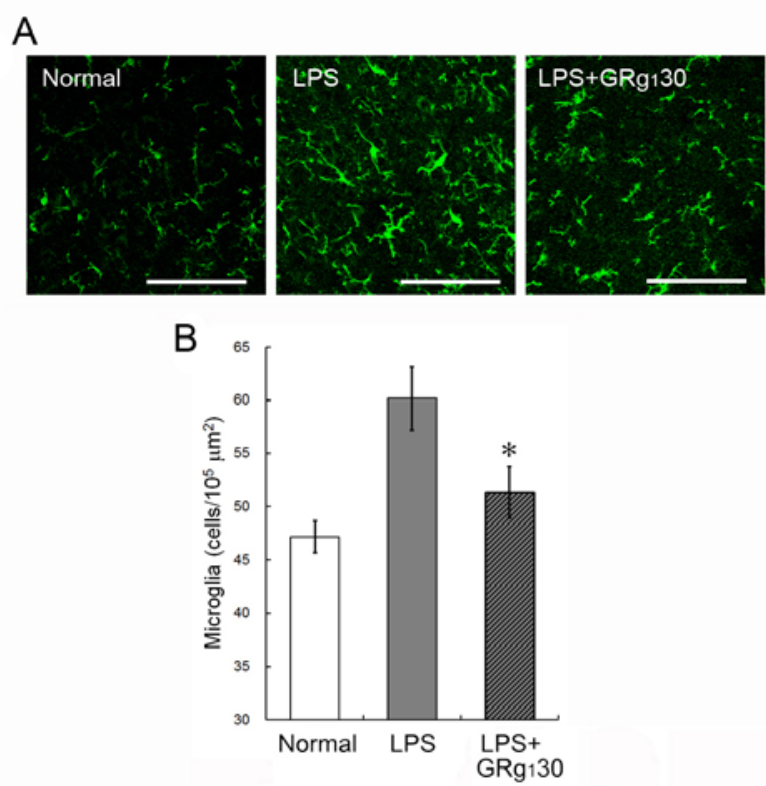

Fig. 4. The effect of $\mathrm{GRg}_{1}$ on microglial activation in the brain. (A) Representative photographs show lba1 immuno-stained microglia in the brain tissue. The LPS group predominantly shows the activated form of microglia, which displays an increased cell size, thickened and shortened processes and intensified lba1 immuno-staining density. The LPS+GRg 130 group shows a decrease in morphological activation of microglia compared to the LPS group. Scale bars are $100 \mu \mathrm{m}$. (B) $\mathrm{GRg}_{1}$ treatment (30 $\mathrm{mg} / \mathrm{kg}$ ) significantly reduced the number of lba1-expressed microglia in the brain. Data are represented by mean \pm SEM ( $n$ $=6$ in each group). Statistical significances are based on comparison to the LPS group $\left(^{*}, \mathrm{p}\langle 0.05)\right.$ using Student' $s$ t-test.
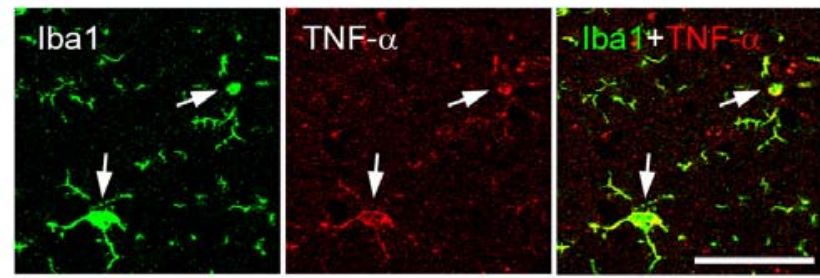

Fig. 5. Representative photographs showing double-immunofluorescent labeling of TNF- $\alpha$ expression with microgla in the brain tissue of LPS-treated mice. TNF- $\alpha$ expression mostly coincides with Iba1-labeled microglia (Iba1+TNF- $\alpha$, arrows). Scale bar is $50 \mu \mathrm{m}$, applicable to all sections.

\section{COX -2 expression in the brain tissue}

The effects of $\mathrm{GRg}_{1}$ on $\mathrm{COX}-2$ expression following systemic LPS injection were tested by quantification of COX -2 protein in the brain tissue at $4 \mathrm{~h}$ after the LPS injection using western blot analysis. LPS increased the percentage of COX-2 expression in the brain tissue $(171.0 \pm 9.8 \%)$, while the $\mathrm{GRg}_{1}$ treatment significantly attenuated the percentage increase of Iba1 expression in the brain tissue at a dose of 30 $\mathrm{mg} / \mathrm{kg}(121.2 \pm 9.4 \%, \mathrm{p}\langle 0.01)$ compared to the LPS group. The brain levels of COX-2 expression in the 10 and $20 \mathrm{mg} / \mathrm{kg} \mathrm{GRg}_{1}$ treatment groups were not significantly different from that of the LPS group 
(Fig. 6).

For a better understanding of the suppressive effect of $\mathrm{GRg}_{1}$ on $\mathrm{COX}-2$ expression, immunohistochemistry against the COX -2 antibody and double-immunofluorescent labeling of COX-2 with the neurons and microglia were performed on the brain tissue. Brain tissue in the normal group exhibited few COX-2-positive cells in the cortex. The LPS group demonstrated a robust increase in COX-2-positive cells in the cortex (Fig. 7, LPS). In contrast, $\mathrm{GRg}_{1}$ treatment reduced the number of COX-2-positive cells in the cortex (Fig. 7, LPS+ $\mathrm{GRg}_{1} 30$ ). Based on the COX-2-positive cell count in the corresponding area, $\mathrm{GRg}_{1}$ treatment significantly reduced the number of COX-2-positive cells in the cortex $\left(23.0 \pm 3.5\right.$ vs. $35.2 \pm 3.2$ cells $\left./ 10^{5} \mu^{2}, p<0.05\right)$ compared with the LPS group (Fig. 7).

In addition, double-immunofluorescent labeling of COX -2 with NeuN or Iba1 identified the properties of the COX-2-positive cells. COX-2-positive cells in the cortex were mostly co-localized with the neurons (Fig. 8 , COX $-2+\mathrm{NeuN})$. Interestingly, $\mathrm{COX}-2$ expression in the cortex did not co-localize with the microglia (Fig. 8 , COX-2+Iba1). This result indicates that $\mathrm{COX}-2$ is mainly expressed on neurons, not on microglia, in the brain tissue following systemic LPS injection.
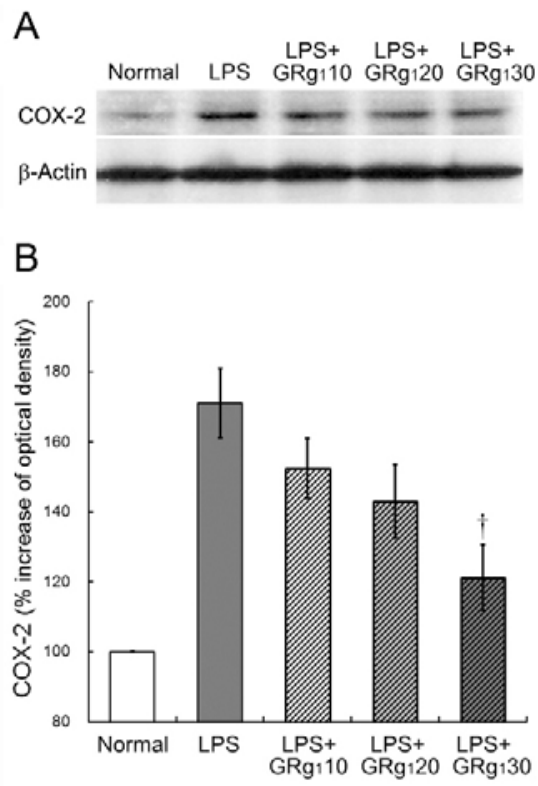

Fig. 6. The effect of $\mathrm{GRg}_{1}$ on COX-2 expression in the brain. (A) Representative western blots illustrate differences in the bands of COX-2. (B) The LPS treatment upregulated COX-2 protein, while $\mathrm{GRg}_{1}$ treatment significantly attenuated upregulation of $\mathrm{COX}-2$ protein at a dose of $30 \mathrm{mg} / \mathrm{kg}$. Data are represented by mean \pm SEM ( $n=6$ in each group). Statistical significances are based on comparison to the LPS group $(\dagger, p<0.01)$ using Student' $s$ t-test.
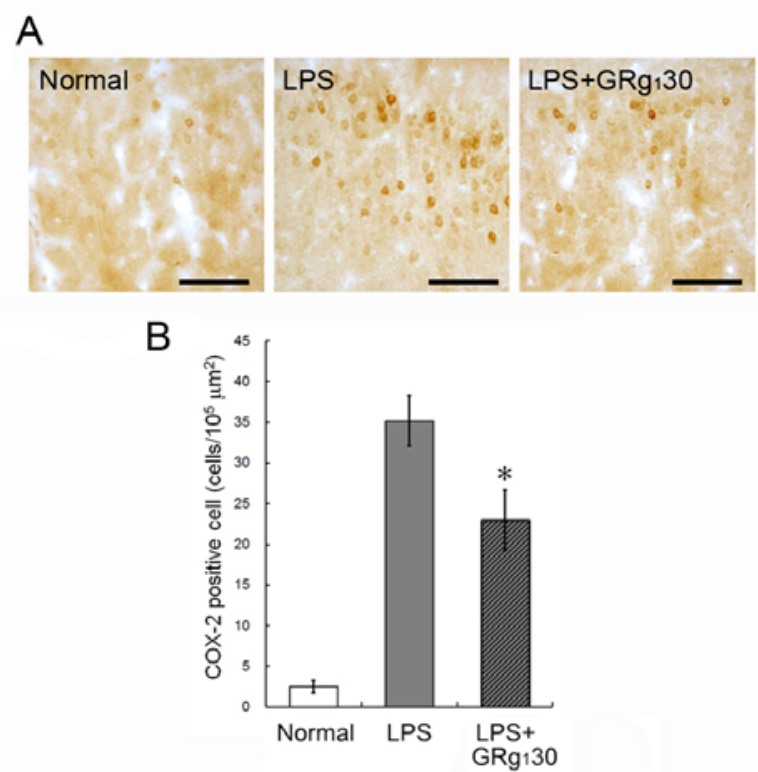

Fig. 7. The effect of $\mathrm{GRg}_{1}$ on COX-2-expressing cells in the brain. (A) Representative photographs show COX-2 immuno-stained cells in the brain tissue. (B) The $\mathrm{GRg}_{1}$ treatment $(30 \mathrm{mg} / \mathrm{kg}$ ) significantly reduced the number of COX-2-expressing cells in the brain. Data are represented by mean \pm SEM $(n=6$ in each group). Statistical significances are based on comparison to the LPS group $\left(^{*}, p<0.05\right)$ using Student' $s$ t-test.
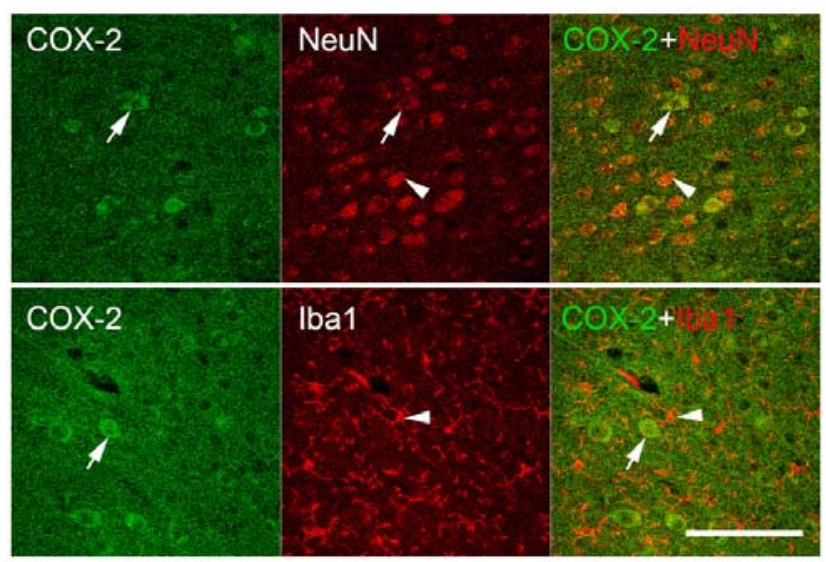

Fig. 8. Representative photographs showing double-immunofluorescent labeling of COX-2 expression with neurons or microgla in the brain tissue of LPS-treated mice. COX-2 expression mostly coincides with NeuN-labeled neurons (COX-2+NeuN, arrows), but not with Iba1-labeled microglia in the brain (COX-2+lba1, arrows and arrowheads). Scale bar is $100 \mu \mathrm{m}$, applicable to all sections.

\section{Discussion}

Systemic LPS treatment (intraperitoneal injection) activates the inflammatory response in the brain and promotes inflammation through the activation of microglia ${ }^{22)}$, and the upregulation of pro-inflammatory cytokines and $\mathrm{COX}-2^{23)}$. In an acute systemic LPS treatment study, inflammatory responses in the brain, including morphological activation of microglia and protein/mRNA expression of inflammatory mediators, appeared within 4-8 hours and subsided within 1-3 
days $^{24)}$. Moreover, Cao et al. reported that induction of COX-2 mRNA in the rat brain via intraperitoneal injection of LPS was most evident in the neuronal cells between 1 and $2.5 \mathrm{~h}$ after injection, and was most evident in non-parenchymal cells between 2.5 and $4 \mathrm{~h}$ after injection ${ }^{25)}$. Thus, in the present study, $\mathrm{GRg}_{1}$ treatment was given once (at a dose of either 10,20 and $30 \mathrm{mg} / \mathrm{kg}$ ) $1 \mathrm{~h}$ prior to the LPS injection. Microglia activation and COX-2 expression were then examined $4 \mathrm{~h}$ after LPS injection.

Microglia, the resident immune cells in the CNS, are activated by an extensive list of pro-inflammatory stimuli, including LPS. Systemic LPS injection activates the inflammatory response in the brain through the toll-like receptors located on glia ${ }^{26)}$. This activates microglia and upregulates pro-inflammatory cytokines, consequently propagating inflammation ${ }^{27)}$. There is abundant evidence suggesting that pro-inflammatory cytokine production and signaling results in neuronal cell death and is closely related to neurodegeneration ${ }^{28,29)}$. In a previous in vitro study of BV-2 microglial cells, $\mathrm{GRg}_{1}$ effectively inhibited $\mathrm{TNF}-\alpha$, IL-1 $\beta$, inducible nitric oxide synthase, and COX-2 via activation of the phospholipase C signaling pathway ${ }^{18)}$. This study showed that $\mathrm{GRg}_{1}$ significantly reduced the over-expression of $\mathrm{TNF}-\alpha$, IL $-1 \beta$, and IL- 6 mRNA in the brain tissue following systemic LPS treatment. This result may expand our understanding of the anti-inflammatory effect of $\mathrm{GRg}_{1}$ on neuroinflammation from in vitro to in vivo. In the present study, GRg 1 significantly attenuated microglia activation in the brain tissue of LPS-treated mice. Microglia are key cellular elements of the acute neuroinflammatory response and are the primary sources of pro-inflammatory cytokines detected in the brain ${ }^{20)}$. A previous report showed that $\mathrm{GRg}_{1}$ attenuated Iba1 expression, which is highly and specifically expressed in microglia and macrophages ${ }^{30)}$, in the cerebral cortex and hippocampus ${ }^{21)}$. The present study demonstrated that $\mathrm{GRg}_{1}$ reduced the morphological change of microglia into the activated form. The activated form shows increased cell size, irregular shape, and thickened and shortened processes in the cerebral cortex. Moreover, $\mathrm{GRg}_{1}$ significantly reduced the number of Iba1-expressing microglia in the cerebral cortex when compared to the LPS group. In addition, $\mathrm{TNF}-\alpha$-expressing cells were identified as microglia by double-immunoflurescent labeling of Iba1 and $\mathrm{TNF}-\alpha$. Overall, the results suggest that $\mathrm{GRg}_{1}$ plays a modulatory role in microglia activation.

COX plays a central role in the inflammatory cascade by converting arachidonic acid into bioactive prostanoids $^{31)}$. COX exists in 2 isoforms: COX-1 and COX-2. COX-1 is a constitutive isoform, which is widely distributed in virtually all cell types and is thought to mediate physiological responses. COX-2 is a second and inducible isoform, which is rapidly expressed in several cell types in response to cytokines and pro-inflammatory molecules and has emerged as the isoform primarily responsible for prostanoid production in acute and chronic inflammatory conditions ${ }^{31,32)}$. Interestingly, excluding a Chinese report by Wang et al. ${ }^{33)}$, there are no in vivo reports about the effect of $\mathrm{GRg}_{1}$ on $\mathrm{COX}-2$ despite its significant role in the modulation of inflammatory responses. As a result of the present study, a dose of $30 \mathrm{mg} / \mathrm{kg}$ of $\mathrm{GRg}_{1}$ significantly attenuated the upregulation of COX-2 mRNA and protein expressions in the brain tissue induced by intraperitoneal LPS injection. Although there are conflicting views about the role of COX-2 in neurodegenerative diseases caused by neuroinflammation ${ }^{31}$, the results of this study suggest that $\mathrm{GRg}_{1}$ might play a role in modulating COX-2 expression induced by LPS. Tthe primary purpose of this study was to show the effect of $\mathrm{GRg}_{1}$ on $\mathrm{COX}-2$ mRNA and protein expressions in the brain tissue. As the results of COX-2 immunohistochemistry in this study show, COX-2 expression was detected in the cerebral cortex. COX-2 expression in the cerebral cortex was attenuated by $\mathrm{GRg}_{1}$ treatment. The COX-2-expressing cells were identified as neurons by double-immunoflurescent labeling of COX-2 and NeuN. Additionally, it was revealed that COX -2 expression did not coincide with Iba1-expressing microglia in the brain tissue of LPS-treated mice. This result confirms that microglia are not the main source of $\mathrm{COX}-2$ production $^{31)}$, even COX-2 expression has been detected in activated microglia preceding neuronal cell death ${ }^{32)}$.

The findings from this study indicate that $\mathrm{GRg}_{1}$ can effectively attenuate COX-2 mRNA and protein expression and microglia activation induced in the brain by systemic LPS treatment. Thus, it is thought that $\mathrm{GRg}_{1}$ and $\mathrm{GRg}_{1}-$ containing Panax ginseng may potentially protect against neurodegenerative diseases caused by neuroinflammation.

\section{Acknowledgement}

This work was supported by a grant (KHU-20110208) from the Kyung Hee University in 2011.

\section{References}

1. Harry GJ, Kraft AD. Neuroinflammation and microglia: 
considerations and approaches for neurotoxicity assessment. Expert Opin Drug Metab Toxicol. 2008 ; $4: 1265-77$.

2. Choi SH, Aid S, Bosetti F. The distinct roles of cyclooxygenase -1 and -2 in neuroinflammation: implications for translational research. Trends Pharmacol Sci. 2009 ; 30 : 174-81.

3. Sparkman NL, Johnson RW. Neuroinflammation associated with aging sensitizes the brain to the effects of infection or stress. Neuroimmunomodulation. $2008 ; 15: 323-30$

4. Nah SY, Kim DH, Rhim H. Ginsenosides: are any of them candidates for drugs acting on the central nervous system?. CNS Drug Rev. 2007 ; 13 : 381404.

5. Attele AS, Wu JA, Yuan CS. Ginseng pharmacology: multiple constituents and multiple actions. Biochem Pharmacol. 1999 ; 58 : 1685-93.

6. Chen LM, Lin ZY, Zhu YG, Lin N, Zhang J, Pan $\mathrm{XD}$, Chen XC. Ginsenoside Rg1 attenuates $\beta$ -amyloid generation via suppressing PPAR $\gamma$-regulated BACE1 activity in N2a-APP695 cells. Eur J Pharmacol. 2012 ; 675 : 15-21.

7. Li W, Chu Y, Zhang L, Yin L, Li L. Ginsenoside Rg1 attenuates tau phosphorylation in SK-N-SH induced by $\mathrm{A} \beta$-stimulated $\mathrm{THP}-1$ supernatant and the involvement of p38 pathway activation. Life Sci. 2012 ; 91 : 809-15.

8. Zhang YF, Fan XJ, Li X, Peng LL, Wang GH, Ke KF, Jiang ZL. Ginsenoside Rg1 protects neurons from hypoxic-ischemic injury possibly by inhibiting Ca2+ influx through NMDA receptors and L-type voltage-dependent Ca2t channels. Eur J Pharmacol. 2008 ; 586 : 90-9.

9. Ge KL, Chen WF, Xie JX, Wong MS. Ginsenoside Rg1 protects against 6-OHDA-induced toxicity in MES23.5 cells via Akt and ERK signaling pathways. J Ethnopharmacol. 2010 ; 127 : 118-23.

10. Zhang X, Wang J, Xing Y, Gong L, Li H, Wu Z, Li Y, Wang J, Wang Y, Dong L, Li S. Effects of ginsenoside $\mathrm{Rg} 1$ or $17 \beta$-estradiol on a cognitively impaired, ovariectomized rat model of Alzheimer's disease. Neuroscience. 2012 ; 220 ：191-200.

11. Fang F, Chen X, Huang T, Lue LF, Luddy JS, Yan SS. Multi-faced neuroprotective effects of Ginsenoside Rg1 in an Alzheimer mouse model. Biochim Biophys Acta. 2012 ; 1822 : 286-92.

12. Shi YQ, Huang TW, Chen LM, Pan XD, Zhang J, Zhu YG, Chen XC. Ginsenoside Rg1 attenuates amyloid-beta content, regulates PKA/CREB activity, and improves cognitive performance in SAMP8 mice. J Alzheimers Dis. 2010 ; 19 : 977-89.

13. $\mathrm{Xu}$ L, Chen WF, Wong MS. Ginsenoside Rg1 protects dopaminergic neurons in a rat model of Parkinson's disease through the IGF-I receptor signalling pathway. Br J Pharmacol. 2009 ; 158 : 738-48.

14. Park SM, Choi MS, Sohn NW, Shin JW. Ginsenoside Rg3 attenuates microglia activation following systemic lipopolysaccharide treatment in mice. Biol Pharm Bull. 2012 ; 35 : 1546-52.

15. Park S, Ahn IS, Kwon DY, Ko BS, Jun WK. Ginsenosides Rb1 and Rg1 suppress triglyceride accumulation in 3T3-L1 adipocytes and enhance beta-cell insulin secretion and viability in Min6 cells via PKA-dependent pathways. Biosci Biotechnol Biochem. 2008 ; 72 : 2815-23.

16. Xu SF, Yu LM, Fan ZH, Wu Q, Yuan Y, Wei Y, Fang N. Improvement of ginsenoside $\mathrm{Rg} 1$ on hematopoietic function in cyclophosphamide-induced myelosuppression mice. Eur J Pharmacol. 2012 ; $695: 7-12$.

17. Lau WS, Chan RY, Guo DA, Wong MS. Ginsenoside Rg1 exerts estrogen-like activities via ligand-independent activation of ERalpha pathway. J Steroid Biochem Mol Biol. 2008 ; 108 : 64-71.

18. Zong $\mathrm{Y}$, Ai QL, Zhong LM, Dai JN, Yang $\mathrm{P}$, He Y, Sun J, Ling EA, Lu D. Ginsenoside Rg1 attenuates lipopolysaccharide-induced inflammatory responses via the phospholipase $\mathrm{C}-\gamma 1$ signaling pathway in murine BV-2 microglial cells. Curr Med Chem. 2012 ; 19 : 770-9.

19. Minghetti L. Cyclooxygenase $-2(C O X-2)$ in inflammatory and degenerative brain diseases. $J$ Neuropathol Exp Neurol. 2004 ; 63 : 901-10.

20. Graeber MB, Streit WJ. Microglia: biology and pathology. Acta Neuropathol. 2010 ; 119 : 89-105.

21. Hu JF, Song XY, Chu SF, Chen J, Ji HJ, Chen XY, Yuan YH, Han N, Zhang JT, Chen NH. Inhibitory effect of ginsenoside $\mathrm{Rg} 1$ on lipopolysaccharide-induced microglial activation in mice. Brain Res. 2011 ; 1374 : 8-14.

22. Henry CJ, Huang Y, Wynne AM, Godbout JP. Peripheral lipopolysaccharide (LPS) challenge promotes microglial hyperactivity in aged mice that is associated with exaggerated induction of both pro-inflammatory IL-1beta and anti-inflammatory IL-10 cytokines. Brain Behav Immun. 2009 ; 23 : 309-17.

23. Chung DW, Yoo KY, Hwang IK, Kim DW, Chung JY, Lee CH, Choi JH, Choi SY, Youn HY, Lee IS, Won $\mathrm{MH}$. Systemic administration of lipopolysaccharide induces cyclooxygenase-2 immunoreactivity in endothelium and increases microglia in the mouse hippocampus. Cell Mol Neurobiol. 2010 ; 30 : 531-41. 
24. Jeong HK, Jou I, Joe EH. Systemic LPS administration induces brain inflammation but not dopaminergic neuronal death in the substantia nigra. Exp Mol Med. 2010 ; 42 : 823-32.

25. Cao C, Matsumura K, Yamagata K, Watanabe Y. Induction by lipopolysaccharide of cyclooxygenase-2 mRNA in rat brain; its possible role in the febrile response. Brain Res. 1995 ; 697 : 187-96.

26. Qin L, Li G, Qian X, Liu Y, Wu X, Liu B, Hong JS, Block ML. Interactive role of the toll-like receptor 4 and reactive oxygen species in LPS-induced microglia activation. Glia. 2005 ; 52 : 78-84.

27. Qin L, Wu X, Block ML, Liu Y, Breese GR, Hong JS, Knapp DJ, Crews FT. Systemic LPS causes chronic neuroinflammation and progressive neurodegeneration. Glia. 2007 ; 55 : 453-62.

28. McAlpine FE, Tansey MG. Neuroinflammation and tumor necrosis factor signaling in the pathophysiology of Alzheimer's disease. J Inflamm Rea. 2008 ; 1 : 29-39

29. Pinteaux E, Trotter P, Simi A. Cell-specific and concentration-dependent actions of interleukin-1 in acute brain inflammation. Cytokine. 2009 ; 45 : $1-7$.

30. Ito D, Imai Y, Ohsawa K, Nakajima K, Fukuuchi Y, Kohsaka S. Microglia-specific localisation of a novel calcium binding protein, Iba1. Brain Res Mol Brain Res. 1998 ; 57 : 1-9.

31. Aid S, Bosetti F. Targeting cyclooxygenases -1 and -2 in neuroinflammation: Therapeutic implications. Biochimie. $2011 ; 93$ : 46-51.

32. Gonzalez-Rey E, Delgado M. Vasoactive intestinal peptide inhibits cyclooxygenase-2 expression in activated macrophages, microglia, and dendritic cells. Brain Behav Immun. 2008 ; 22 : 35-41.

33. Wang Q, Zheng H, Zhang ZF, Zhang YX. Ginsenoside Rg1 modulates COX-2 expression in the substantia nigra of mice with MPTP-induced Parkinson disease through the P38 signaling pathway. Nan Fang Yi Ke Da Xue Xue Bao. 2008 ; 28 : 1594-8. 